\title{
IMPROVING THE EFFICIENCY OF A PHOTOVOLTAIC SYSTEM BY INCORPORATING TRACKING SYSTEM AND MPPT: A REVIEW
}

UDC ((621.311.243+621.472):004.41.421MPPT)

\author{
S. Udhayakumar, R. A. Sindhu, R. Srivasthan, Y. Yogaraj \\ Department of Mechanical Engineering, PSG College of Technology, Coimbatore,
} India

\begin{abstract}
The harvesting of solar energy is gaining increasing attention as it is pollution free and is available in abundance. Various researches and experiments are being carried out to improve the efficiency of power conversion by altering the material of the photovoltaic panels, by incorporating tracking systems and by making use of Maximum Power Point Tracking (MPPT) algorithms. The conventional rigidly fixed solar panels limit their area of exposure to the sun during the entire day. The use of tracker increases the area of panel exposed to direct beam of the sun, thus increasing the power generated. MPPT algorithm tracks the maximum power point attained at all loads and extracts the power from the panel at that voltage. Despite the variations in the external environment, the power obtained from the panel is always maximum. This paper reviews various tracking methods and MPPT techniques to increase the energy harvesting capacity of the panel and in turn improve its efficiency.
\end{abstract}

Key words: photovoltaics, MPPT, solar tracker

\section{INTRODUCTION}

With the ever increasing demand for energy and declining availability of conventional resources, there has been an increase in the focus of improving the energy obtained through renewable resources. These renewable resources comprise hydro power, wind energy, biofuel, solar energy etc. Hydro and wind energy are unpredictable in nature and the production of biofuel requires biomass and a conversion unit. The ubiquitous nature of solar energy makes it the most preferred renewable energy to focus on. Solar power is

Received August 16, 2017

Corresponding author: R A. Sindhu

PSG College of Technology, Department of Mechanical Engineering, Coimbatore, India

E-mail: milan.dincic@elfak.ni.ac.rs 
harnessed by the use of photovoltaic systems, concentrated solar power and solar water heating systems. The annual potential of solar energy is more than 1575 EJ [1]. The current use of the available solar power is about 0.1 EJ [1]. Fig. 1 shows the amount of annual solar irradiance on the surface of the earth.

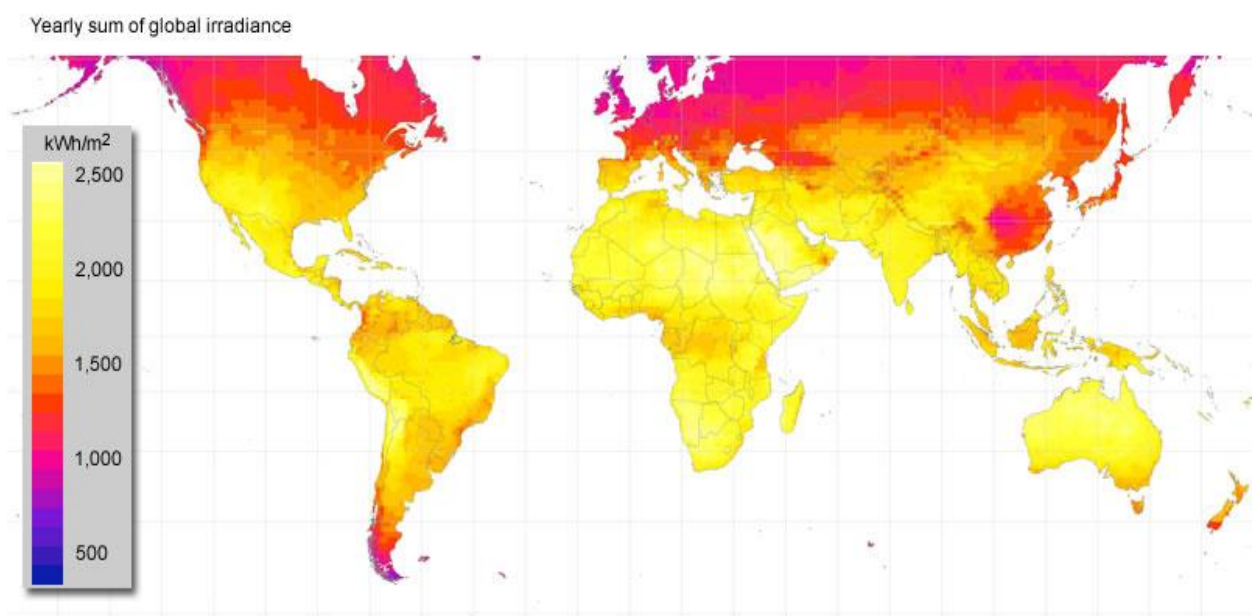

Fig. 1 Global annual solar irradiance

Source: www.greenrhinoenergy.com

In order to increase the utilization of the available solar energy the area of land involved in solar power collection is to be increased. The allocation of large areas of land for installation of solar plants is futile when the power conversion efficiency of the system is low. Thus more focus is to be on improving the efficiency of the solar power harnessing system. The energy conversion efficiency of the system depends on the conversion circuit, weather conditions, incident angle of solar irradiance etc. Various techniques have been implemented to maximize the energy obtained from these systems. The techniques involve the shifting of the system's orientation at periodic intervals during the year, the use of a single or dual axis tracking system and the use of Maximum Power Point Tracking (MPPT) algorithms. A brief study has been done on the various available techniques tested and implemented on solar photovoltaic cells.

\section{SOLAR TRACKING}

Maximum solar radiation is incident on a photovoltaic panel when it is placed at right angles to the sun's rays. To ensure perpendicular incidence of solar rays on the solar panel, incorporating a tracking system in the solar panel is proposed. The solar incidence on a panel is characterized by three angles, azimuth angle, elevation angle and incidence angle as shown in Fig. 2. 


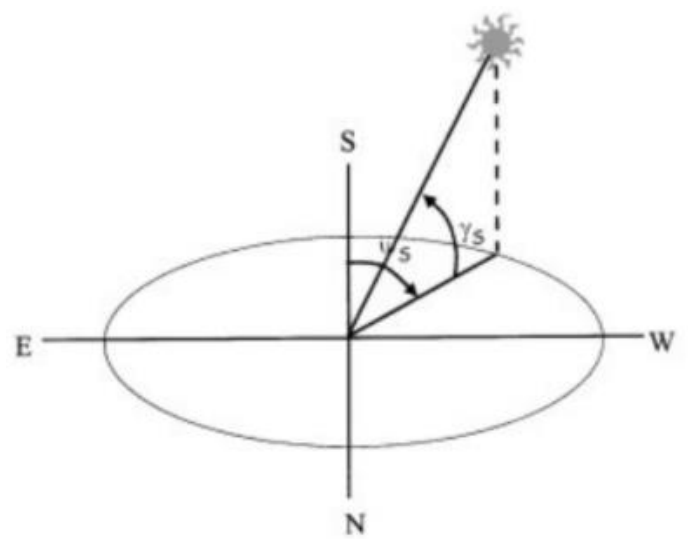

(a)

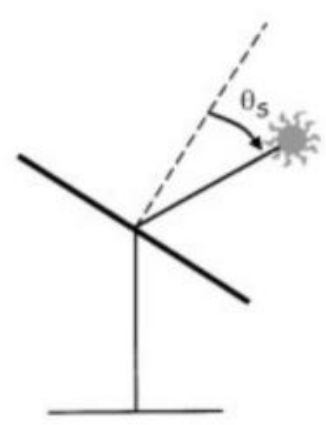

(b)

Fig. 2 (a) Solar coordinates: azimuth $\psi_{\mathrm{s}}$ and elevation $\gamma_{\mathrm{s}}$; (b) incidence angle, $\theta_{\mathrm{s}}$ [3]

Solar tracking is done in two ways, with or without the use of a sensing system to detect the position of the sun.

\subsection{By the use of equations}

Formulas that describe the position of the sun at different latitudes have been framed for azimuth and elevation angles [4]. The solar tracking formulas of the azimuth-elevation method with $\theta_{H}$ as the elevation angle and $\rho_{H}$ as the azimuth angle are given in equations (1) and (2). Fig 3 shows the orientation of the solar panel after it has been rotated.

$$
\begin{gathered}
\theta_{H}=\frac{\pi}{2}-\arcsin \left(\frac{-\sin \lambda+\cos \Phi \cos \delta \cos \omega+\sin \Phi \sin \delta}{2 \cos \theta_{i}}\right) \\
\rho_{H}=\arcsin \left(\frac{\cos \lambda \sin \phi-\cos \delta \sin \omega}{2 \cos \theta_{i} \cos \beta_{H}}\right)
\end{gathered}
$$

where

$$
\begin{aligned}
\theta_{i}=0.5 \arccos \{ & (\sin \Phi \cos \delta \cos \omega-\cos \Phi \sin \delta)(-\cos \lambda \cos \phi) \\
& -(\cos \delta \sin \omega \cos \lambda \sin \phi)-\sin \lambda(\cos \Phi \cos \delta \cos \omega+\sin \phi \sin \delta)\}
\end{aligned}
$$

$\phi$ is the rotation angle about zenith from north towards east.

$\lambda$ is the altitude angle of target relative to the earth's surface.

$\Phi$ is the local latitude

$\delta$ is the declination angle of the sun

$\omega$ is the solar hour angle. 


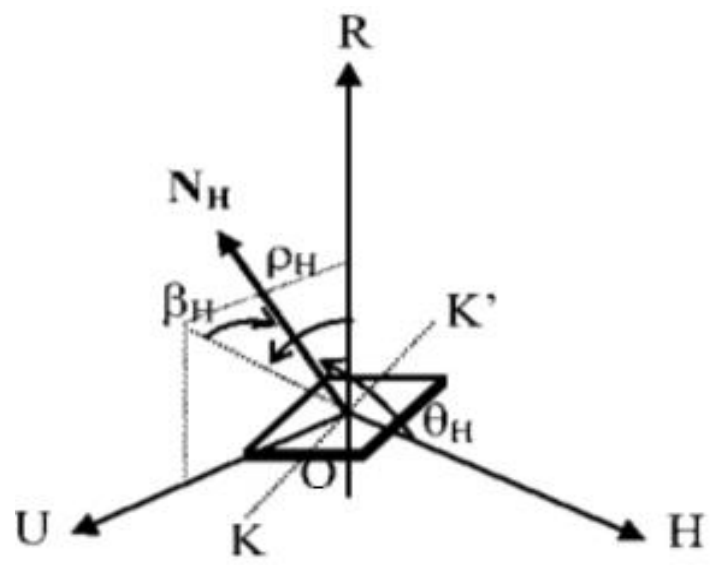

Fig. 3 Position of the panel oriented to the tracking position after rotation [4]

Chen, Lim and Lim [4] presented a most general form of solar tracking formula for a heliostat that is arbitrarily oriented towards an arbitrarily located target. The previous existing tracking equations include the azimuth-elevation tracking formulae and the further developed spinning-elevation sun tracking formula. The authors derived a general form of sun tracking formula from which the previously existing tracking methods can be extracted as special conditions.

An offline sensorless dual-axis solar tracker was designed by Fathabaldi [14] for photovoltaic systems and solar concentrators. The data required for tracking were extracted from solar map equations. The proposed system was cost effective as it did not require any feedback signal or sensor. The operation was independent from external disturbances and weather conditions. An increase of about $20 \%$ was observed in the absorbed solar energy and the tracking error of the system was found to be $0.43^{\circ}$.

\subsection{By the use of a sensing system}

The use of a sensing system for the detection of the position of maximum irradiation involves the use of light detecting sensors and a control unit. The light detecting sensor generally used is the light detecting resistor $[5,6]$. Two light detecting resistors are used per axis. Al-Mohamad [6] made use of two LDR for sensing along the azimuth axis. A Programmable Logic Controller (PLC) unit was used as the control unit to operate the drive system of the tracker. Bingol, Altintas and Oner [5] designed a dual-axis tracker using four LDR and a microcontroller.

Though the use of a sensing unit adds to the cost of the tracker, it is more preferred compared with the system involving solar equations. The solar radiation is not constant throughout the year and varies with altitude and location. These variations are not accommodated in the equations. 


\subsection{Hybrid systems}

An attempt was made by Rubio et al [7] to integrate the two modes of solar tracking. A hybrid tracking system consisting of solar movement model based open loop tracking strategies and dynamic feedback controller based closed loop strategies was proposed. Two tracking modes were programmed in the system based on the detected solar radiation. The normal tracking mode was used when the tracking error was small and the absorbed solar radiation was high. The search mode was used when there was a hitch in the solar tracking using normal mode. When the tracking error is large, no energy is produced in the cell. In the search mode, the structure moved along a square spiral in the azimuth-elevation place to detect the position of the sun. Once the position was attained, the controller shifted to normal tracking mode. The proposed hybrid system was found to be more effective than the classical open loop tracking strategy. Fig. 4 shows the operation of the hybrid system where $u$ represents the position of the panel and $y$ is the estimated position of the sun.

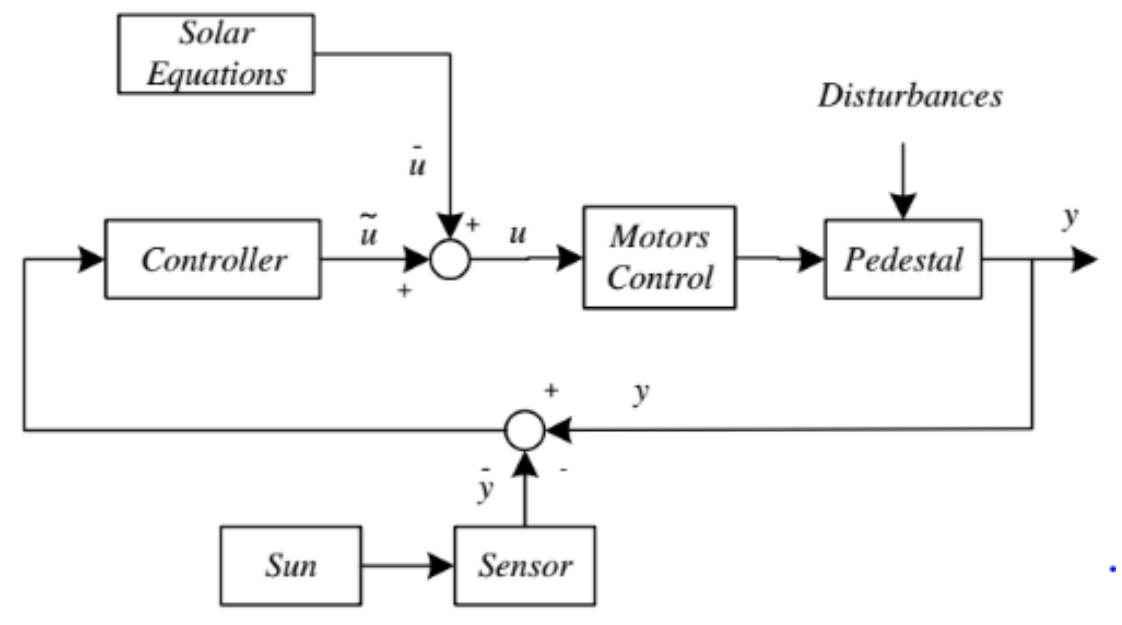

Fig. 4 Operation of the hybrid system [7]

A dual-axis tracker with four photo detectors was designed by Roth, Georgiev and Boudinov [15]. A computer was used to control two DC motors to orient the panel. If the sensors are unable to detect the sun, such as while the sun is covered by clouds, the position of the sun is calculated by a computer program to orient the panel until the detector could sense the sun's position. The computer program makes use of the existing solar tracking formulas to determine the position of the sun.

\subsection{Single-axis tracking}

Single-axis solar trackers involve the movement of the panel about a single-axis, usually the azimuth axis. The panel may have a manual elevation adjustment on the second axis which can be adjusted throughout the year at regular intervals. The single-axis systems are 
preferred for small photovoltaic power plants as the system involves only one rotation axis. The presence of a single rotation axis instead of two reduces the number of moving components and the requirement of complex control systems. Fig. 5 shows a single-axis tracking system comprising a tilted PV panel and a drive mechanism to move the panel on an approximate trajectory relative to the sun's position.

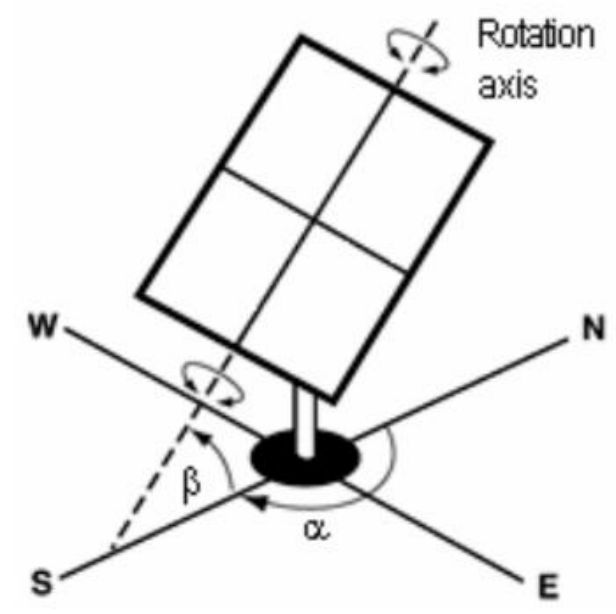

Fig. 5 Single-axis solar tracker [8]

Tudorache and Kreindler [8] executed a single-axis solar tracking device for a photovoltaic conversion panel to assess its performance. The tracker has been designed by making use of two light sensors mounted normal to the panel separated by an opaque plate. Based on the difference in the detected signals, the movement of the solar panel is decided. This ensures that the panel moves for a significant change in light intensity preventing intermittent, frequent and unnecessary movements. It was concluded that the proposed design was advantageous when compared to fixed PV conversion panels.

A single-axis solar tracking system with two small solar panels facing each other in a triangular setup was designed by Rizk and Chaiko [9]. The panel movement was dependent on the difference in the detected voltage from the small panels. A power increase of $30 \%$ was observed with respect to a fixed horizontal array. Saravanan, Panneerselvam and Christopher [10] designed a low cost tracking system that uses the sun as its guiding source instead of taking the earth as the reference. The panel movement was controlled by the circuit based on the signal received from the sensors. The proposed system was observed to attain an increase of 25 to $30 \%$ in energy conversion at low cost and minimum maintenance. Poulek and Libra [2] designed a solar tracker using a bifacial solar cell connected to a DC motor. The arrangement was made use of in bifacial solar arrays with the tracking panel perpendicular to the array.

The effect of using a single-axis solar tracking system in large-grid connected photovoltaic plant was studied by Lorenzo et al [3]. The study was carried out in Tudela in a plant of capacity 1.4 MW. The tracking system was examined taking into account the effects of shadowing between the trackers and the back-tracking features. It was observed 
that azimuth tracking resulted in an increase in energy gain of up to $40 \%$ when compared with optimally tilted static arrays. Vieira et al [12] performed a comparative study between a static photovoltaic solar panel and a mobile panel fitted with single-axis solar tracker. The study was carried out in Mossoro, Brazil, near the equator where there is no large variation in the position of the sun throughout the day. The study showed an increase in the generated power in the mobile panel when compared to the static panel.

Song et al [11] designed a single axis tracking device that lifts and lowers the photovoltaic panels based on the previously available data without the use of a sensing system. The proposed design consisted of a bidirectional sliding axle for solar azimuth tracking. Mounting of the panel on the slide way enabled the control of gravitational center of the panel. The design allowed the fixing of the panel horizontal to the building surface when strong winds were encountered. The simple assembling, easy installation on building, wind resistance and light weight of the system made it suitable for installation on roof-tops and for building integration.

\subsection{Error characterization}

To determine the precision of a solar tracker, a testing procedure was proposed by Sallaberry et al [13]. A parabolic trough collector was tested to estimate the positioning angle error. A digital inclinometer was used to characterize the angular tracking error. The method proposed by the Spanish committee was verified experimentally for a singleaxis tracker. Though the proposed method focused on a small-size parabolic trough collector (PTC), it could be applied to a general single-axis solar tracker.

\subsection{Dual-axis tracking}

Dual-axis tracking involves the rotation of the solar panel about two perpendicular axes. Thus, the system has two degrees of freedom, namely rotation about the azimuth axis and rotation about the elevation axis. The dual-axis tracker is more accurate than a single-axis tracker as the variations along the elevation angle is taken care of. Since the dual-axis tracking system involves sensing along two axes, a complex control system is used. Fig. 6 shows the two axis position control of the solar panel.

Bingol, Altintas and Oner [5] designed a dual-axis tracker with the sensors placed inside four hollow cylindrical tubes. The assembly was mounted on the solar panel making $45^{\circ}$ with the panel plane. The difference in the observed light intensity was taken as the criterion for operating the stepper motor to move the panel towards the direction of the

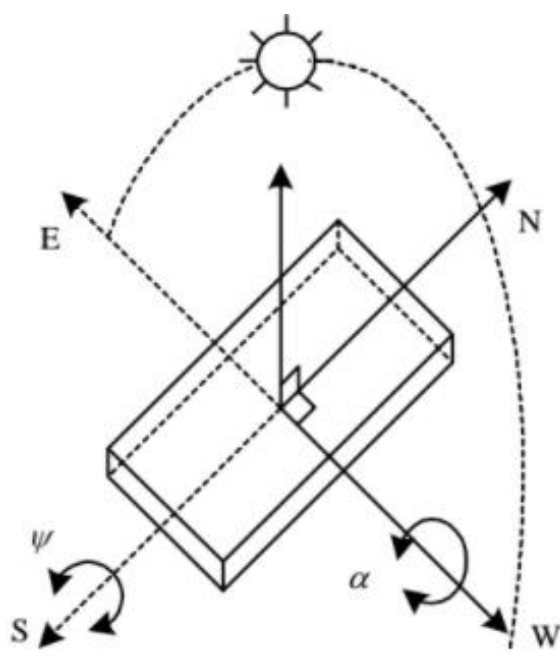

Fig. 6 Two axis position control of the solar panel [5] sun. Kacira et al [16] studied the effects of two-axis solar tracker on photovoltaic panels with respect to fixed panels in Turkey. A gain of $34.6 \%$ in the generated power was 
attained with the use of two-axis solar tracking solar panel when compared to a south facing panel at $14^{\circ}$ tilt angle.

Patil et al designed, fabricated and tested a two-axis equator based solar tracking mechanism controlled using computer. The design of the system was such that the operation of the tracker consumed a very small fraction of the generated power. The structural components were designed taking into consideration the self-weight, wind forces and the imposed load due to tracking motion. The equator based tracking algorithm was fed to the system and it was installed and tested for tracking errors. The correction factors were added and the system was found to have a maximum deviation of $3^{\circ}$ over a day. The observed increase in the output of the photovoltaic module was about $30 \%$.

Wu, Zhang and Wang [20] proposed a two-axis sun-tracking mechanism. The U-3PSS (Universal - 3 Prismatic Spherical Spherical joint) solar tracker was a redundant design while U-2PSS was its nonredundant counterpart. The trackers were tested for workspace and energy consumption to determine the optimum design. The study found that the U-3PSS tracker had a bigger workspace and smaller energy consumption than the U-2PSS tracker.

A dual-axis tracking system was developed for photovoltaic conversion panels in Ourgla city by Khadidja et al [17]. Tracking PV modules result in an increase in yield of $15 \%$ to over $35 \%$ when compared to fixed mounted PV installations. A two-axis tracking system has been developed and mounted on the PV panels and the solar insolation, power generated and the panel efficiency were calculated for the entire day and conclusion were drawn from the results. Though generating the tracking trajectories for a system is complex, the tracking system reduces the losses incurred due to shading. Yao et al [18] developed dual-axis tracking system with two strategies. The normal tracking mode ensured low tracking errors while the daily adjustment mode altered the primary axis once a day. The former strategy yielded an increase of $23.6 \%$ and the latter strategy showed an increase of $31.8 \%$ in the efficiency of the panel.

Mi et al [21] proposed a solar tracking strategy that utilizes an open-loop mode to control and alter the tracking frequency of the system. This proved to be more efficient than the conventional tracking system using constant tracking frequency. High concentrating photovoltaics require accurate tracking system for high performance. Increasing tracking frequency increased efficiency as well as moving frequency of the system and mechanical wear. Thus varying tracking frequency strategy was introduced. The program for controlling the tracking frequency was written based on the theoretical solar velocity in different time and date. The use of the proposed system reduced the moving frequency of the tracking system which saved the energy required to drive the system and reduced mechanical wear.

\subsection{Lateral translations}

The conventional method of solar tracking involves the rotating of the entire panel assembly to maintain direct normal illumination on the panel from the sun. An alternative approach to the tilting system is to move the photovoltaic device relative to an array of lenses placed over an array of small photovoltaic cells. Hallas et al [19] proposed demonstrated two-axis tracking in high-concentration solar power optics using a lenslet array over a planar waveguide. The planar micro-optic solar concentrator was designed with a lenslet array over a planar waveguide with small reflective facets at the focal point of each 
lenslet. This aids in coupling the incident light into the waveguide. The tracking of solar power was carried out by lateral translation of the lenslet with respect to the waveguide. It was observed that about $75 \%$ of incident energy was collected on using a refracted doublet in conjunction with a polar tracker. The system was able to successfully track the Sun in spite of cloud cover. Fig. 7 shows the illustration of planar micro-optic solar concentrator operation.

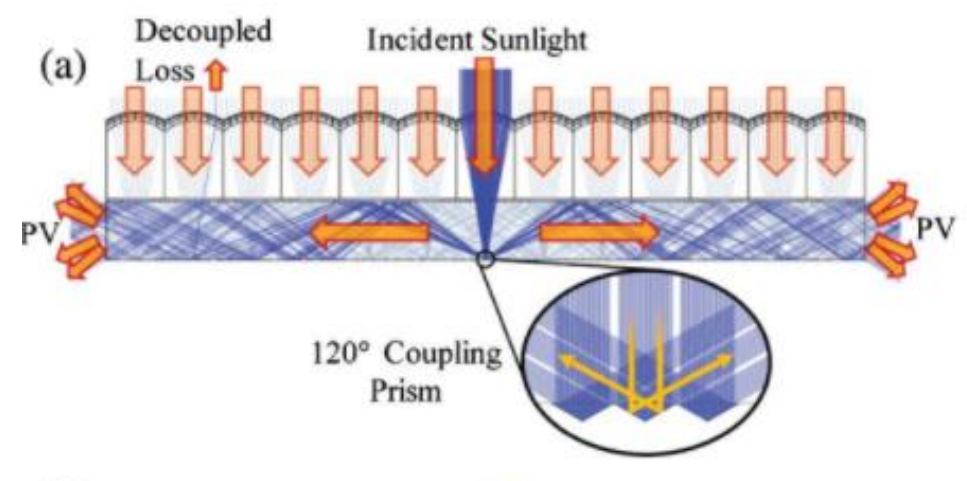

(b)

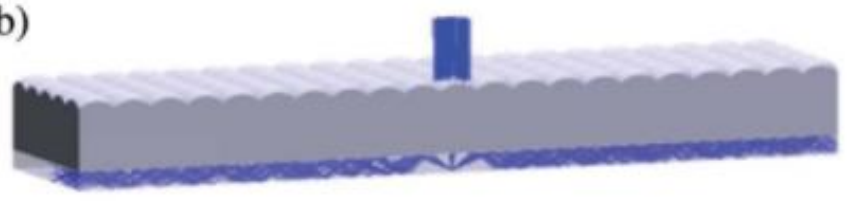

Fig. 7 (a) Lenslets and microprisms direct sunlight into a slab of waveguide with edge mounted photovoltaic cells; (b) perspective view with one lenslet illuminated [19]

The advantages of this system involve easy motion control as it requires only motion in one plane, rigidity to wind load due to a fixed outer frame and compact form factor. The drawback of using the system is the requirement of a large number of small photovoltaic cells, packaging tolerances and cost.

\subsection{Comparison of tracking systems}

The various solar panels available for use are the fixed panel, the panel with vertical axis tracking, the panel with eat-west axis tracking, the panel with north-south axis tracking and the panel with dual-axis tracking. Abdallah [22] investigated the effect of all five types of panels on the power generated in flat plate photovoltaics. A power gain of about $40 \%$ was observed when two-axis tracker was used when compared with a fixed surface inclined $32^{\circ}$ to the south in Jordan. Vermaak [24] analysed the cost and energy produced from the different solar tracking configurations in different scenarios. It was observed that dual axis tracking was more efficient than single axis tracking and fixed panels for small photovoltaic systems. For large plants, the use of tracking system proved to be inefficient as the number of modules in the given region of land was less compared to static modules. 
Bahrami, Okoye and Atikol [23] examined the performance of various solar trackers at different latitudes by studying the hourly solar radiation data from locations around Europe and Africa. It was observed that different solar trackers exhibited different energy gain with the change in latitude. Thus it was concluded that the tracking performance of the different trackers were highly dependent on the location of installation. The study was done to aid the designers in the selection of appropriate solar tracker system based on the latitude of the location. Table 1 shows the effect of latitude on the ranking of the trackers.

Table 1 Effect of latitude on the ranking of the trackers [23]

\begin{tabular}{|c|c|c|c|c|c|c|c|c|}
\hline S.No. & CATEGORY & & & OBSERVE & RANKIN & & & \\
\hline 1 & $0<$ lat. $<6$ & Dual axis $>\mid E W O>$ & IEW > & $E W>$ & VO > & $V F>$ & NS > & Fixed \\
\hline 2 & $6<$ lat. $<26$ & Dual axis > IEWO > & $\mid E W>$ & VO > & $E W>$ & VF $>$ & NS > & Fixed \\
\hline 3 & $26<$ lat. $<57$ & Dual axis > V0 > & |EWO > & $\mid E W>$ & $V F>$ & $E W>$ & NS > & Fixed \\
\hline 4 & $57<$ lat. $<64$ & Dual axis > VO > & |EWO > & $\mid E W>$ & $V F>$ & NS > & $E W>$ & Fixed \\
\hline 5 & lat. $>64$ & Dual axis $>$ VO > & $V F>$ & IEWO > & IEW > & NS $>$ & Fixed $>$ & EW \\
\hline
\end{tabular}

\section{MAXIMUM POWER POINT TRACKING}

The output from the photovoltaic cell is affected by the changes in solar irradiance and cell temperature. In order to extract maximum power from the cell under varying loads and atmospheric conditions, Maximum Power Point Tracking (MPPT) controllers are to be provided. The incorporation of MPPT in the photovoltaic conversion circuit reduces the number of solar panels required to extract the desired output power.

The electrical characteristics of a solar cell are nonlinear which lowers the effectiveness of extracting energy in non-stationary environments. The photovoltaic module is to operate at peak power for improved efficiency. When the photovoltaic array is directly connected to a load, the operating point of the system will be at the intersection of the current-voltage curve of the array and the load line [28]. At most times, this operating point is not the module's maximum power point. Thus an oversized array must be used to obtain the required power from the module. In order to obtain the maximum power from the system irrespective of the disturbances, MPPT system is used. The photovoltaic source terminal voltage is effectively controlled such that the product of voltage and current achieves a maximum value. This maximum value is called maximum power point and the point at which it is attained is known as the knee of the PV diode normal current-voltage curve [29].

Goudar, Patil and Kumar [28] reviewed the algorithms available to track the maximum point of a photovoltaic module. The various algorithms for tracking the maximum power point are open-circuit voltage method, perturb and observe algorithm (P\&O), Optimized $\mathrm{P} \& \mathrm{O}$ algorithm, variable step incremental conductance algorithm, parasitic capacitance algorithm, fuzzy logic control, neural network.

Mohapatra et al [31] studied a number of maximum power point tracking algorithms that would prove effective for solar panels under partial shading conditions. The solar panels that do not have a tracker are not normal to the solar incidence radiation throughout the day. Thus during the course of the day, the panel is subjected to varying degrees of irradiance due 
to the movement of the earth and shading conditions due to the placement of solar panels. Hybrid algorithms, new modelling approach and converter topologies have been introduced to track the maximum power point.

Alsadi and Alsayid [32] proposed a system to simulate the maximum power point tracking using the perturb and observe algorithm for photovoltaic systems. Matlab/Simulink was used for establishing a model of the photovoltaic system with MPPT function. This model was combined with the solar PV module and the DC-DC Boost converter models. Different climatic conditions were simulated and evaluated. It was found that the system tracked the maximum power point accurately.

Alippi and Galperti [30] proposed a low-power MPPT circuit for wireless sensorless nodes. The system was found to be effective in critical weather conditions such as when the cell was in shadow or when the weather was cloudy. Mirbagheri, Mekhilef and Mirhassani [34] introduced maximum power point tracker with incremental conductance method in a one-diode model. Two topologies were selected for testing and were compared. The conventional interleaved boost converter proved to be more efficient and faster than the conventional boost converter.

Kumar, Bhaskar and Koti [35] compared the incremental conductance maximum power point tracking algorithm used in solar array power systems with the existing direct control method. The proposed method eliminated the use of proportional integral control loop method and proved to be more efficient and accurate than the short circuit current MPPT method especially at varying temperature.

Kim, Kin and Youn [33] proposed a maximum power point tracker for a photovoltaic system without the use of a solar array current sensor. The information on solar array current was obtained from a sliding-mode observer. As the estimated solar array current had some chattering ripples, it was averaged over a half cycle of the alternating current utility grid frequency with the measured solar array voltage. Through simulation and experiments, it was observed that the system provided a superior performance compared to the conventional system and eliminated the use of an expensive current sensor.

\section{CONCLUSION}

The present technology provides a clean and inexpensive form of conversion of solar energy into electrical energy by the use of photovoltaic systems. Recent studies have shown that the use of solar tracking system increased the energy produced in the photovoltaic modules. The use of hybrid tracking systems which made use of sensors and solar equations proved to be efficient at all weather conditions. The use of Maximum Power Point Tracking (MPPT) system along with the conversion circuit ensured the extraction of the desired power from the module and eliminated the use of an oversized array of solar panels. The incorporation of tracking system and MPPT increased the efficiency of power conversion of the solar panel. This increases the amount of electrical energy produced without having to erect additional solar panels to obtain the desired energy levels and thus reducing the cost of electricity. 


\section{REFERENCES}

[1] World Energy Assessment: Energy and the Challenge of Sustainability, United Nations Development Programme, 2000

[2] Poulek V., Libra M., "A very simple solar tracker for space and terrestrial applications", Solar Energy Materials and Solar Cells, vol. 60, 2000, pp $99-103$.

[3] Lorenzo E., Perez M., Ezpleta A., Acedo J., "Design of Tracking Photovoltaic Systems with a Single Vertical Axis", Progress in Photovoltaics: Research and Applications, 2002, pp 533-543.

[4] Chen Y. T., Lim B. H., Lim C. S., "General Sun Tracking Formula for Heliostats with Arbitrarily Oriented Axes", Journal of Solar Engineering, vol 128, 2006, pp $245-250$.

[5] Okan Bingol, Ahmet Altintas, Yusuf Oner, "Microcontroller Based Solar Tracking System and its Implementation", Journal of Engineering Sciences, 2006, pp 243 - 248.

[6] Ali Al-Mohamad, "Efficiency improvements of photo-voltaic panels using a Sun-tracking system", Applied Energy, vol 79, 2004, pp $345-354$.

[7] Rubio F.R., Ortega M.G., Gordillo F., Lopez-Martnez M., "Application of New Control Strategy for Sun Tracking", Energy Conversion and Management, vol 48, 2007, pp 2174 - 2184

[8] Tudorache Tiberiu, Kreindler Liviu, "Design of a Solar Tracker System for PV Power Plants", Acta Polytechnica Hugarica, vol 7, 2010, pp 23 - 39.

[9] Rizk J., Chaiko Y., "Solar Tracking System: More Efficient Use of Solar Panels", World Academy of Science, Engineering and Technology, vol 41, 2008, pp $313-315$.

[10] Saravanan C., Panneerselvam M.A., Christopher William I., "A Novel Low Cost Automatic Solar Tracking System”, International Journal of Computer Applications, vol 31, 2011, pp 62 - 67.

[11] Song Jifeng, Zhu Yong, Xia Dancheng, Yang Yongping, "A Photovoltaic Solar Tracking System with Bidirectional Sliding Axle for Building Integration”, Energy Procedia, vol 61, 2014, pp $1638-1641$.

[12] Viera R.G., Guerra F.K.O.M.V., Vale M.R.B.G., Araujo M.M., "Comparative Performance Analysis between Static Solar Panels And Single Axis Tracking System on a Hot Climate Region Near to the Equator", Renewable and Sustainable Energy Reviews, vol 64, 2016, pp 672 - 681.

[13] Sallaberry Fabienne, Pujol-Nadal Ramon, Larcher Marco, Rittmann-Frank Mercedes Hannelore, "Direct Tracking Error Characterization on a Single-Axis Solar Tracker", Energy Conversion and Management, 2015, pp $1281-1290$

[14] Fathabadi Hassan, "Novel High Efficient Offline Sensorless Dual-Axis Solar Tracker for Using in Photovoltaic System and Solar Concentrator", Renewable Energy, vol 95, 2016, pp 485 - 494

[15] Roth P., Georgiev A., Boudinov H., "Design and Construction of a System for Sun Tracking", Renewable Energy, vol 29, 2004, pp 393 - 402.

[16] Kacira Murat, Simsek Mehmet, Babur Yunus, Demirkol Sedat, "Determining Optimum Tilt Angles and Orientations of Photovoltaic Panels in Sanliurfa, Turkey”, Renewable Energy, vol 29, 2004, pp 1265 1275 .

[17] Khadidja Bouziane, Dris Korichi, Boubeker Azoui, Nourreddine Settou, "Optimization of a Solar Tracker System for Photovoltaic Power Plants in Saharian Region, Example of Ouargla", Energy Procedia, vol 50, 2014, pp $610-618$.

[18] Yao Yingxue, Hu Yeguang, Gao Shengdong, Yang Gang, Du Jinguang, "A Multipurpose Dual-Axis Solar Tracker with Two Tracking Strategies", Renewable Energy, vol 71, 2014, pp 88 - 98.

[19] Hallas Justin M., Baker Katherine A., Karp Jason H., Tremblay Eric J., Ford Joseph E., "Two-axis Solar Tracking Accomplished through Small Lateral Translations", Optical Society of America, vol 51, 2012, pp $6117-6124$

[20] Wu Jun, Zhang Binbin, Wang Liping, "Optimum Design and Performance Comparison of a Redundantly Actuated Solar Tracker and its Nonredundant Counterpart", Solar Energy vol 127, 2016, pp $36-47$.

[21] Mi Zhe, Chen Jikun, Chen Nuofu, Bai Yiming, Fu Rui, Liu Hu, "Open-loop Solar Tracking Strategy for High Concentrating Photovoltaic systems using Variable Tracking Frequency", Energy Conversion and Management, vol 117, 2016, pp $142-149$

[22] Abdallah Salah, "The Effect of Using Sun Tracking Systems on the Voltage-Current Characteristics and Power Generation of Flat Plate Photovoltaics", Energy Conversion and Management, vol 45, 2004, pp $1671-1679$.

[23] Bahrami Arian, Okoye Chiemeka Onyeka, Atikol Ugur, "The Effect of Latitude on the Performance of Different Solar Trackers in Europe and Africa", Applied Energy, vol 177, 2016, pp 896 - 906. 
[24] Vermaak H.J., "Techno-economic Analysis of Solar Tracking Systems in South Africa", Energy Procedia, vol 61, 2014, pp 2435 - 2438

[25] Sumathi Vijayan, Jayapragash R., Bakshi Abhinav, Akella Praveen Kumar, "Solar Tracking Methods to Maximum PV System Output - A Review of Methods Adopted in Recent Decade", Renewable and Sustainable Energy Reviews, vol 74, 2017, pp 130 - 138

[26] Patil J.V., Nayak J.K., Sundersingh V.P., "Design, Fabrication and preliminary Testing of a Two-axes Solar Tracking System”, RERIC International Journal, vol 19, 1997, pp 15 - 23.

[27] Eldin Sharaf S.A., Abd-Elhady M.S., Kandil H.A., "Feasibility of Solar Tracking Systems for PV Panels in Hot and Cold Regions", Renewable Energy, vol 85, 2016, pp 228 - 233.

[28] Goudar M.D., Patil B.P., Kumar V., "A Review of Improved Maximum Peak Power Tracking Algorithms for Photovoltaic Systems", International Journal Electrical Engineering and Technology, 2010, pp $85-107$.

[29] Zhou Z., Holland P.M., Igic P., "MPPT Algorithm Test on Photovoltaic Emulating System Constructed by a DC Power Supply and an Indoor Solar Panel”, Energy Conversion and Management, vol 85, 2014, pp $460-469$

[30] Alippi Cesare, Galperti Cristian, “An Adaptive System for Optimal Solar Energy Harvesting in Wireless Sensor Network Nodes", IEEE Transactions on Circuits and Systems, vol 55, 2008, pp 1742 - 1750

[31] Mohapatra Alivarani, Nayak Byamakesh, Das Priti, Mohanty Kanungo Barada, “A Review on MPPT Techniques of PV System under Partial Shading Conditions", Renewable and Sustainable Energy Reviews, vol 80, 2017, pp $854-867$.

[32] Alsadi Samer, Alsayid Basim, "Maximum Power Point Tracking Simulation for Photovoltaic Systems using Perturb and Observe Algorithm", International Journal of Engineering and Innovative Technology, vol 2, 2012, pp $80-85$.

[33] Kim Il-Song, Kim Myung-Bok, Youn Myung-Joong, "New Maximum Power Point Tracking Using Sliding-Mode Observer for Estimation of Solar Array Current in the Grid-Connected Photovoltaic System", IEE Transaction on Industrial Electronics, vol 53, 2006, pp 1027 - 1035.

[34] Mirbagheri Zahra S., Mekhilef Saad, Mirhassani Mohsen S., "MPPT with Inc. Cond Method using Conventional Interleaved Boost Converter", Energy Procedia, vol 42, 2013, pp $24-32$.

[35] Kumar Kalyan K., Bhaskar R., Koti Hemanth, "Implementation of MPPT Algorithm for Solar Photovoltaic Cell by Comparing Short-circuit Method and Incremental Conductance Method", Procedia Technology, vol 12, 2014, pp $705-715$.

[36] Singh, "Solar Power Generation by PV (Photovoltaic) Technology: A Review", Energy, vol 53, 2013, pp $1-13$. 